\title{
Modelo de Desarrollo Turístico Sostenible para cantones costeros: herramienta para actores locales de Manabí, Ecuador
}

\section{Model of Sustainable Tourism Development for coastal cantons: Tool for local actors from Manabí, Ecuador}

\author{
William Patricio Proaño Ponce ${ }^{1}$ \\ Jorge Freddy Ramírez Pérez ${ }^{2}$ \\ Universidad Pinar del Río, Cuba
}

\begin{abstract}
Resumen. El Ecuador como territorio vive tiempos decisivos en su historia, por ejemplo: la actividad turística se convierte en un componente esencial de acción integrada en territorios locales. Igualmente, el impulso de esta actividad provoca beneficios y deterioros en cantones costeros, descubre actores locales con apreciación compleja respecto a beneficios provenientes del turismo. Además, se evidencia debilidad de gobiernos locales en solventar las necesidades turísticas colectivas, las cuales tienen tiempos de respuesta prolongados. Por lo tanto, se efectúa una investigación exploratoria, deductiva, descriptiva; se emplea triangulación de resultados logrados de fuentes de información primaria y secundaria, con el objetivo de precisar datos ambientales, socioculturales, económicos, políticos, institucionales y elementos que connotan la gestión del desarrollo turístico sostenible en cantones costeros de la provincia Manabí, Ecuador. Bajo el diseño de planificación ligada a características particulares de cada territorio, se selecciona cantones costeros con mayor concentración y atractivos turísticos en la provincia Manabí, que permiten encontrar respuesta a los objetivos planteados. Desde los fundamentos teóricos y metodológicos, resulta un modelo único en la gestión para el desarrollo turístico sostenible en cantones costeros que responde al contexto de su territorio y corresponda un proceso de adaptación hacia el destino. La idea de diseñar este modelo es hacerlo como "traje a la medida" y no bosquejar una producción de modelos en serie.
\end{abstract}

Palabras clave. Desarrollo Turístico Sostenible, cantones costeros, actores locales

\begin{abstract}
Ecuador as a territory lives decisive times in its history, tourism becomes an essential component of integrated action in local territories. The boost of this activity brings benefits and deterioration in coastal cantons, discovers local actors with full appreciation of benefits from tourism, and there is evidence of the weakness of local governments in responding to collective tourism needs, which have long response times. An exploratory, deductive, descriptive research is carried out using triangulation of results obtained from primary and secondary information sources, with the objective of specifying the environmental, socio-cultural, economic, political and institutional data and elements that connote the management of sustainable tourism development in coastal cantons of the province of Manabí, Ecuador. Under the planning design linked to the particular characteristics of each territory, coastal cantons with greater concentration and tourist attractions are selected in the province of Manabí, which allow finding a response to the objectives pursued. From the theoretical and methodological foundations, a unique model results in the management for the sustainable tourism development in the coastal cantons that respond to the context of their territory and correspond to a process of adaptation towards the destination. The idea of designing this model is to do it as a "tailor made suit" and not to sketch a production of serial models.
\end{abstract}

Keywords. Sustainable Tourism Development, Coastal Cantons, Local Actors

${ }^{1}$ William Patricio Proaño Ponce, doctorante, Facultad de Economía y Empresariales, Universidad Pinar del Río, Pinar del Río - Cuba. Correo electrónico: mantawilliam@yahoo.com

²Jorge Freddy Ramírez Pérez. Profesor Centro de Estudio, Gerencia, Desarrollo Local y Turismo (GEDELTUR), Universidad Pinar del Río, Pinar del Río Cuba. Correo electrónico: freddy@upr.edu.cu 


\section{Introducción}

El artículo constituye un avance de los resultados en la investigación sobre la gestión para el desarrollo turístico sostenible en cantones costeros del sur de la provincia Manabí, Ecuador; se describe el modelo conceptual y la metodología que se utiliza en la caracterización de los territorios donde se aplicará. Además, se provee como herramienta para gestionar un desarrollo turístico sostenible en los espacios costeros, cuyo actor protagónico es el gobierno autónomo descentralizado. Dicho actor debe basarse en el aprovechamiento de los recursos endógenosy exógenos, a fin de diseñar estrategias integradas orientadas a la sostenibilidad del desarrollo local.

A partir de estudios sobre fundamentación teórica y metodológica del diseño de modelos, se muestra un modelo impar en la gestión para el desarrollo turístico sostenible en cantones costeros, el cual corresponde a un proceso de adaptación hacia la economía local, mancomunidad, entre otros elementos particulares del destino. En definitiva, la idea de diseñar este modelo es hacerlo único y no esbozar una producción de modelos en serie.

La implementación del modelo establece oportunidad para el desarrollo turístico sostenible en cantones costeros del territorio estudiado, debido a la vinculación y a la articulación institucional entre políticas económicas, sociales y ambientales. A la vez, este supone la articulación de medios apropiados y que se convierta en una herramienta hacia los actores locales de los cantones costeros del sur de la provincia Manabí.

El presente modelo se construye con orientación al buen vivir ${ }^{3}$ y en armonía con la

\footnotetext{
3 "Buen Vivir" o "Vivir Bien" es una extrapolación del concepto Kichwa del Sumak Kawsay "Es la forma de vida que permite la felicidad y la permanencia de la diversidad cultural y ambiental; es armonía, igualdad, equidad y solidaridad. No es buscar la opulencia ni el crecimiento económico infinito".- PNBV, 2013, p.14.
}

naturaleza, donde el ser humano es sujeto y fin de esfuerzos por el desarrollo. Al mismo tiempo, se conjuga con las dimensiones sustantivas de la sostenibilidad (ambientales, económicas, sociales y político institucional).

Por medio de triangulación con resultados obtenidos de las fuentes de información, primarias y secundarias, permite establecer precisiones relacionadas con el proceso de gestión para el desarrollo turístico sostenible local en los cantones costeros de la provincia Manabí, Ecuador. También, se demuestra la necesidad de integrar y de fortalecer la autonomía y descentralización como distinción de los gobiernos locales.

Un aspecto clave es involucrar a todos los actores locales, sean estos públicos, privados o de economía popular y solidaria, donde cada uno debe adquirir las siguientes capacidades: valorizar el entorno, formarse para trabajar con un carácter mancomunado con otros sectores $\mathrm{y}$, con esto, mantener relaciones con otros cantones costeros del territorio ensayado. El conjunto de estas capacidades se correlaciona con el enfoque propuesto en el modelo, el cual es el desarrollo turístico sostenible.

\section{Metodología}

La investigación se desarrolló en la zona costera de los distritos Bahía Ballena y Puerto Cortés hasta el pie de monte, definido en la cota 20 m.s.n.m, para un área de estudio total de 12 223.10 ha , entre los $9^{\circ} 16^{\prime}$ Norte, $83^{\circ} 50^{\prime}$ Oeste y $\operatorname{los} 8^{\circ} 7^{\prime}$ Norte, $83^{\circ} 28^{\prime}$ Oeste.

Los actores locales inmersos en el estudio se conformaron por representantes de asociaciones, organizaciones, presidentes barriales, miembros de entidades barriales, entre otros, que contribuyeron en la elaboración de los Planes de Desarrollo y Ordenamiento Territorial (PD y OT), de los cantones costeros de la provincia Manabí. El Instituto Nacional de Estadísticas 
y Censos (INEC, 2016), así como los PD y OT de cada cantón costero de esta provincia, permitió, durante el periodo 2016-2017, tener información sobre el número de actores involucrados en la gestión de esos territorios.

Para determinar el tamaño de la muestra en la aplicación de encuestas hacia actores afines a la actividad turística, se utilizaron criterios de Pérez (2013) y Calero (1976); se empleó el muestreo irrestricto aleatorio, con una confiabilidad de $95 \%$, una proporción de 0,50 (que permite el mayor tamaño de muestra) y un error de muestreo de 0,05. Para datos cualitativos, se hizo una selección de una muestra necesaria de 320, en los cantones costeros del sur de Manabí, de acuerdo con los procedimientos de cálculos utilizados por los autores citados, según la siguiente formula:

$$
n=\frac{\left(\frac{Z_{1-\alpha / 2}}{d}\right)^{2} P(1-P)}{1+\frac{1}{N}\left(\frac{Z_{1-\alpha / 2}}{d}\right)^{2} P(1-P)-\frac{1}{N}}
$$

\section{Dónde:}

P: es la proporción de individuos (la n máxima se obtiene cuando $p=0,5$ )

1- $\alpha$ : confiabilidad

d: error absoluto

Con una confiabilidad de $.95 \mathrm{Z1}-\alpha / 2=1,96$ un error absoluto de .05 con $p=.5$ y N $=1898$ con un tamaño de muestra necesario de 319,64 $\approx 320$

La información se logró mediante recolección de datos a través de las técnicas siguientes: encuesta, entrevistas, análisis documental.
Esto permitió identificar y comprender las característicasdecantones costerosenla provincia Manabí. Las fuentes primarias ilustraron el comportamiento en la gestión de la actividad turística, el análisis de contribución en políticas actuales para el desarrollo turístico, los criterios en gestión de procesos que se desarrollan en las costas de Manabí; la percepción sobre el turismo y el desarrollo a escala local, entre otros.

Los datos de fuentes secundarias permitieron la clasificación del territorio por caracterizar, el cual se precisó en función de las potencialidades especiales, tales como reservas ecológicas o atractivos turísticos. Esto se cumplió mediante la revisión de documentos oficiales, el método deductivo, la investigación descriptiva, con lo cual se logró detallar, analizar e interpretar la descripción actual de los cantones costeros de la provincia Manabí. No obstante, para analizar de manera más amplia y llegar a conclusiones, se utilizó el software Statistical Package For Social Sciences (SPSS), versión 22.0 español, el cual facilitó procesar, con cualidad estadística, los resultados de las técnicas utilizadas.

\section{Resultados}

\section{Precisión del territorio}

Ecuador es un país ribereño, que tiene supremacía oceánica, posee el Archipiélago de Galápagos y, según el Ministerio del Ambiente de Ecuador (MAE, 2014), le extiende las millas náuticas en 4,3 veces su área continental. Esto expresado en distancia se determina en 1111818 $\mathrm{km}^{2}$; en este sentido, los beneficiarios directos para el desarrollo de una actividad serían actores locales de las áreas costeras, que se estiman de acuerdo con el MAE (2014) en 3900000 habitantes en toda la zona costera de Ecuador, esto incluye 600000 pescadores artesanales pertenecientes a 168 caletas y puertos pesqueros.

En Ecuador, el Código Orgánico de Organización Territorial Autonomía Descentralización 
(COOTAD, 2011) establece la división política administrativa en regiones, provincias, cantones y parroquias, como jurisdicciones territoriales con Gobiernos Autónomos Descentralizados (GAD). Sobre el sistema de planificación, se liga a características particulares del territorio que se busca desarrollar y ordenar. En este contexto, la Secretaría Nacional de Planificación y Desarrollo (SENPLADES, 2010) crea nueve zonas que agrupan a las 24 provincias del país y a 4 cantones. Tal es así, que cada GAD debe crear PD y OT de acuerdo con los lineamientos y las directrices de estrategia territorial nacional y de las agendas zonales respectivas.

Con el propósito de fortalecer y de mejorar la articulación entre niveles de gobierno, el Plan Nacional para el Buen Vivir (PNBV, 2013) expresa que el gobierno nacional inició procesos de desconcentración y de descentralización. Para ese efecto, se creó una nueva forma administrativa de planificación del estado ecuatoriano conformada por nueve zonas (figura 1), esimportante destacar quelas agendas zonales

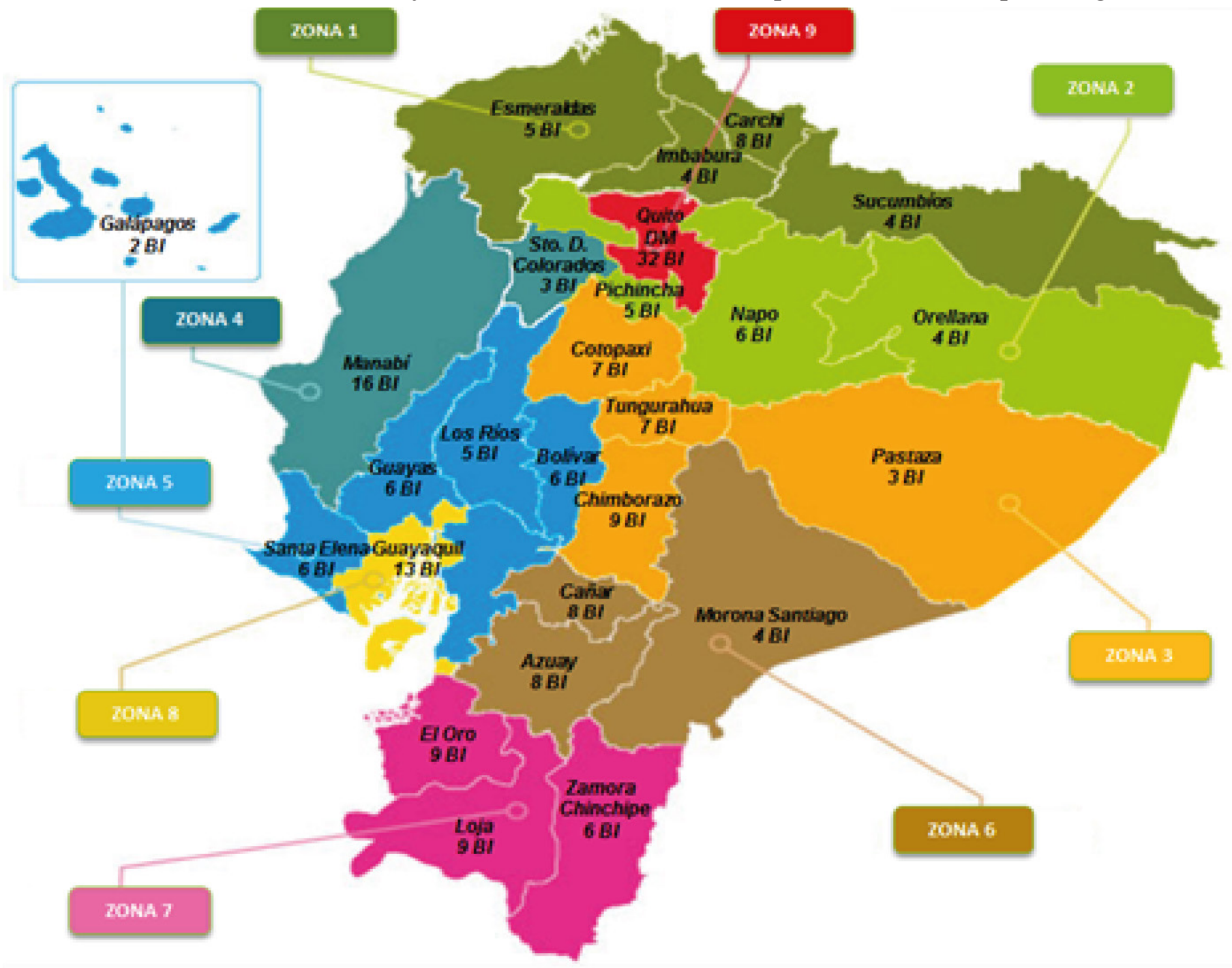

Figura 1. Mapa Ecuador: división zonas de planificación y político territorial. Fuente: Agenda Zonal 4 Pacífico 2013-2017 (SENPLADES, 2015). 
no alteran la división político-administrativa. Por tanto, los niveles de gobierno conservan su autonomía y gobernabilidad.

En este contexto, se expresa que una zona está conformada por dos o más provincias con continuidad territorial; además, debe tener una superficie mayor a $20000 \mathrm{~km} 2$ y un número de habitantes que, en su conjunto, sea superior a $5 \%$ de la población nacional.

Sobre el turismo en Ecuador, el COOTAD instituye que, en un cantón, la primera autoridad del GAD se denomina alcalde. Entre sus funciones se hallan : regular, controlar y promover el desarrollo de la actividad turística local. También, le corresponde establecer el departamento encargado de esta actividad, además de proveer espacios de organizaciones asociativas y de empresas comunitarias de turismo, así como fomentar la inversión y el desarrollo económico, especialmente, de la economía popular y solidaria.

La Zona 4 Pacífico (figura 2) tiene una superficie de 22 717,82 km2, la cual corresponde al 8,73\% de la superficie del país. En esta área, existe una población de 1072233 de habitantes en el área urbana; y 708 484, en el área rural, lo que equivale a $12,29 \%$ de la población total, según el Instituto Nacional de Estadísticas y Censos (INEC, 2010), de los cuales 50,21\% corresponden a los hombres y $49,79 \%$, a mujeres.

Ahora bien, destaca 60,21\% de la población que reside en áreas urbanas y 39,79\% en áreas rurales.

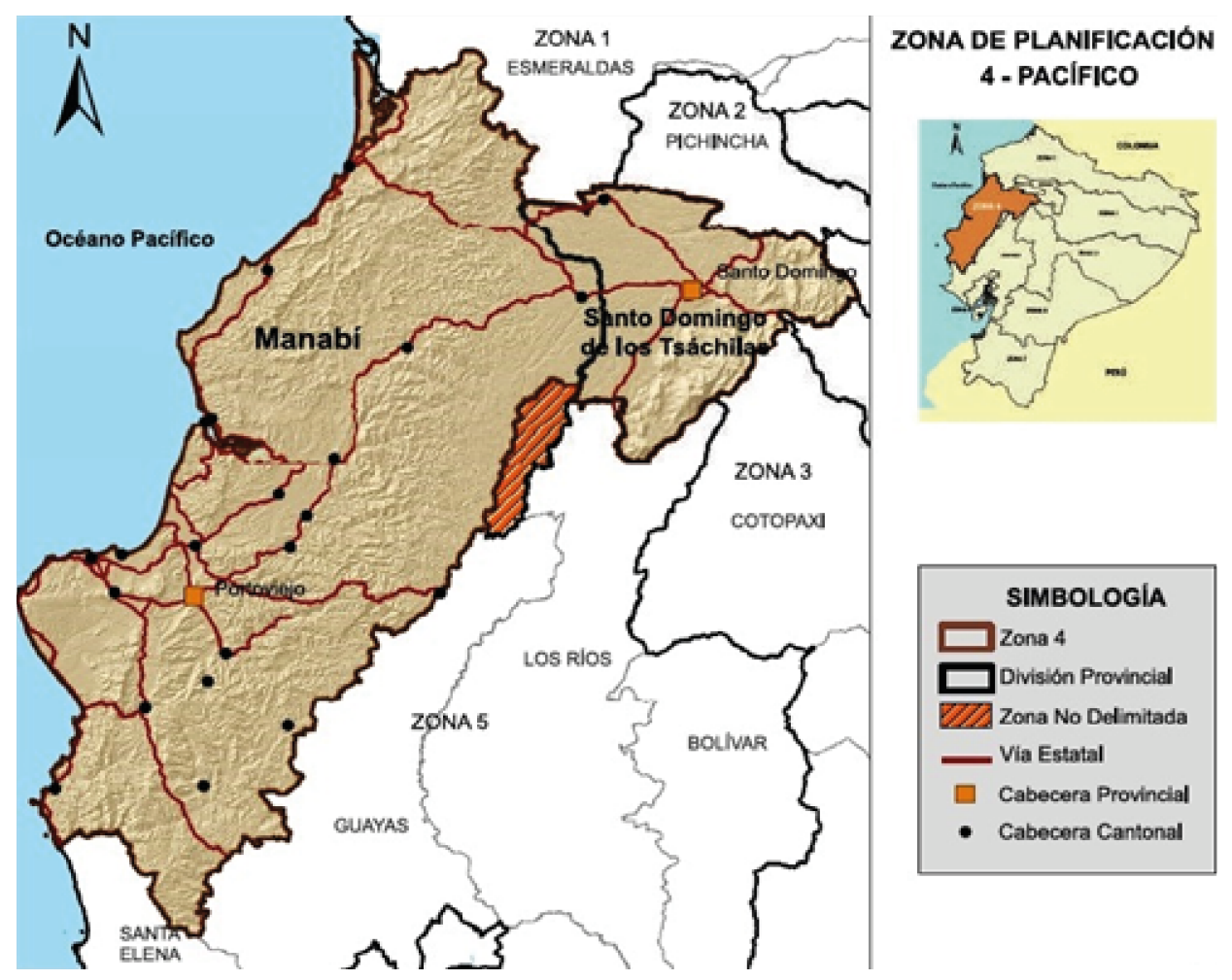

Figura 2. Mapa de Zona 4-Pacífico, limitación y delimitación. Fuente: Agenda Zonal 4 Pacífico 2013-2017 (SENPLADES, 2015). 
En este sentido, la densidad poblacional es de $78,38 \mathrm{hab} / \mathrm{km} 2$, cifra superior a la del país, que es de $56 \mathrm{hab} / \mathrm{km} 2$; la tasa de crecimiento poblacional es de $1,77 \%$, de los cuales, la provincia de Manabí es de 1,6\% y la de Santo Domingo de los Tsáchilas es de $2,36 \%$. Respecto a la distribución de la población comprendida entre los 0 a 24 años, representa 51,38\%. Esta lectura revela que la población es mayoritariamente joven, lo cual significa un gran potencial para el desarrollo.

La provincia de Manabí posee todo el perfil costero de la Zona 4 Pacífico que cuenta con una longitud aproximada de $370,53 \mathrm{~km}$, según la Secretaria Técnica del Mar (SETEMAR, 2014). Además, esta provincia agrupa22 cantones y cuenta con una tasa de crecimiento poblacional del $1,60 \%$.

Los núcleos de población jerarquizados que forman el eje industrial y comercial se integran en los cantones del sur, como Manta, Montecristi, Jaramijó, Portoviejo (capital), Santa Ana, Rocafuerte, Jipijapa y Puerto López, cantones que generan flujos comerciales y turísticos de acuerdo con lo determinado por las regulaciones de la SENPLADES (2015).

En esta misma orientación, se encuentran cantones en la parte norte, como Bahía de Caráquez, San Vicente, Pedernales, Chone, Calceta, Tosagua, donde predominan las actividades agropecuarias y después las turísticas que, a manera de nodo articulador, la capital de la provincia de Santo Domingo de los Tsáchilas dinamiza el eje comercial entre estos cantones.

\section{Realidad turística}

Desde el ámbito nacional, el Ministerio de Turismo (MINTUR, 2014) establece que Ecuador es uno de los pueblos con mayor biodiversidad del planeta; además, la ha mantenido durante los últimos años. El INEC (2016) refleja ese incremento de la actividad con la llegada de turistas debido a las maravillas naturales que posee: en 2010, con una entrada de extranjeros de 1047 098; en el 2016, ingresaron 1412 718 visitantes extranjeros, observándose un incremento significativo de 34,92\%. Sin embargo, hubo un decrecimiento en el año $2015 \quad(-0,8 \%)$ y en el $2016(-8,53 \%)$, por causas de depreciación en la moneda de países vecinos, Colombia y Perú, además de las graves consecuencias que dejó el terremoto acaecido en las costas ecuatorianas el 16 de abril de 2016.

Para el presente diagnóstico, se contó con instrumentos oficiales y leyes del estado ecuatoriano, así como el criterio de diversos actores claves inmersos en la problemática investigada, entre los documentos están: Constitución de la República de Ecuador, PNBV, COOTAD, Agenda Zonal 4-Pacífico, Código Orgánico del Ambiente, Plan Nacional de Control y Vigilancia, INEC, además de los PD y OT de cantones costeros de la provincia Manabí. Entre las precisiones que se consideraron para el análisis de la realidad turística costera, están:

- Principios del Plan Nacional para el Buen Vivir (PNBV) de Ecuador, que se constituye en la hoja de ruta de la actuación pública para construir los derechos de las personas y que genera la transformación en el país.

- Relaciones productivas que permitan definir el desarrollo de funciones vinculadas con actividades comerciales y prestación de servicios. Están delimitadas por la agenda zonal 4 Pacífico.

- Tendencias en la personalización como factor de diferencia en el desarrollo de una actividad turística.

- Tendencia en traslados por rutas hacia espaciosnaturales, patrimoniales, gastronómicos, demanda caracterizada por la OMT y el Instituto de Promoción de Exportaciones e Inversiones (PRO ECUADOR, 2014).

- Plan estratégico de desarrollo de turismo sostenible para Ecuador. 
Sobre la investigación procesada relativa a cantones costeros, se enfatizó el contexto ambiental, económico, sociocultural, políticoinstitucional. Tal es así que reveló problemáticas en la gestión para el desarrollo turístico, las cuales obstaculizan situar esta actividad como alternativa de desarrollo; estas son:

- Los usos y la ocupación del suelo en la franja costera están sometidos a presión de diversas fuentes: contaminación terrestre, extracción excesiva de los recursos naturales, crecimiento acelerado de la población costera.

- La inequidad y la debilidad en la calidad de los servicios turísticos.

- Los servicios de alojamiento y la alimentación muestran problemas de certificación de calidad y de cobertura territorial.

- La insuficiente fiscalización y regulación en las actividades turísticas.

- Los operadores turísticos y las agencias de viaje evidencian baja propensión a la innovación en su oferta de productos.

- Las actividades legalmente constituidas, como las turísticas, se ven enfrentadas a una dura competencia de precios con un sector informal nada despreciable.

- La desarticulación entre niveles de gobierno es una debilidad latente, debido a que los PD y OT exhiben gestiones opuestas.

- La falta de aplicación de la planificación en los gobiernos anteriores conllevan a múltiples problemas de carácter social. Todavía no cubren las necesidades básicas insatisfechas y otros servicios.

- La separación entre los objetivos de desarrollo y la realidad del territorio; se desconocen las potencialidades y las limitaciones para alcanzar el desarrollo propuesto.
- El desconocimiento de la efectividad de las acciones de control y de vigilancia de las áreas marino costeras protegidas de Ecuador continental.

Por lo expuesto sobre cantones costeros del sur de la provincia Manabí, y basado en los documentos citados, se demuestra que aun cuando existe la voluntad política del Gobierno central ecuatoriano para potenciar el desarrollo turístico en las costas ecuatorianas de este territorio. En cambio, la gestión de los gobiernos autónomos descentralizados de los cantones involucrados, en la presente investigación, son insuficientes para la explotación de potencialidades que contribuyan a posicionar al turismo como una opción para el desarrollo de esta actividad con enfoque sostenible.

Elautor agrupa cantones costeros como proceso fundamental de planificación. En este sentido, Alcántara (2014) expone que toda congregación territorial o zonificación turística debe identificar y priorizar los recursos, así como delimitar, de manera espacial, el área de desarrollo turístico para establecer políticas locales.

La figura 3 muestra la concentración de turismo en Manabí, el autor considera esta agrupación por tener mayor relevancia para él estudio.

Respecto al proceso de gestión turística en Ecuador, los aportes del Ministerio de Turismo (MINTUR, 2007) expresan que toma fuerza a partir dela creación deldiseñodel Plan Estratégico de Desarrollo de Turismo Sostenible para Ecuador (PLANDETUR-2020) que surgió con el fin de preservar y de conservar la biodiversidad de recursos reales y potenciales del país. Este modelo de gestión nacional considera tres ejes: sostenibilidad, competitividad, fortalecimiento del marco legal e institucional.

\section{Sostenibilidad en el turismo costero}

El turismo sostenible contribuye a un desarrollo económico y de progreso sociocultural, el cual 


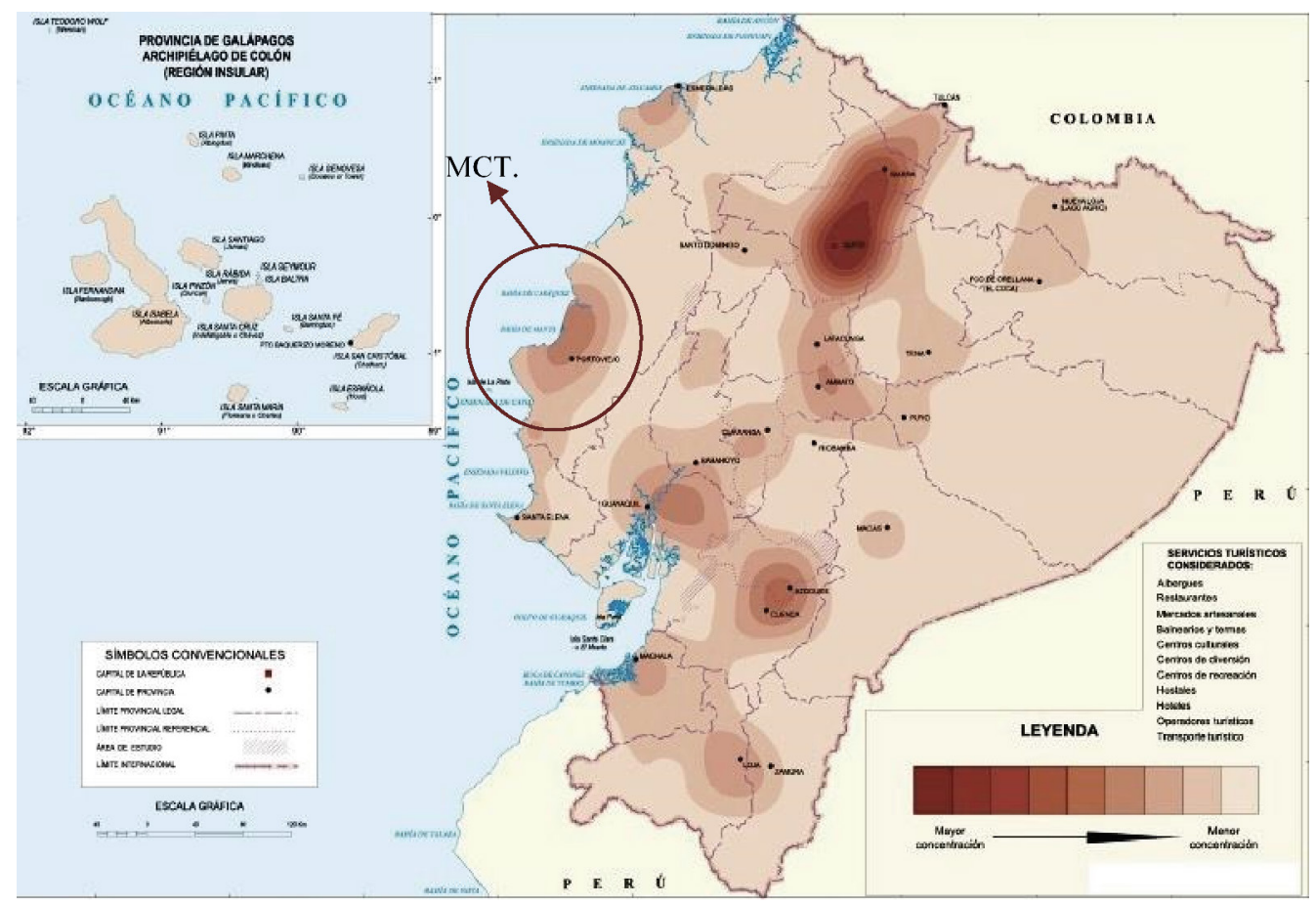

Figura 3. Concentración de servicios turísticos. Leyenda: MCT, mayor concentración de turismo en costas del sur de Manabí. Fuente: Atlas Instituto Geográfico Militar (IGM, 2014).

requiere de una gobernanza involucrada para fortalecerlo. Al mismo tiempo, se requiere un uso adecuado de recursos naturales y que se produzca en armonía con el entorno; todo esto debe crear el equilibrio entre las dimensiones sustantivas de la sostenibilidad, ajustándose a las necesidades presentes y a la conservación de los mismos recursos turísticos para el futuro.

En este orden de ideas, se debe tomar en cuenta que al turismo sostenible no se le debe considerar una elección, sino que se debe asumir como compromiso de todos los actores involucrados en la actividad turística. Si bien el turismo costero ha mejorado, no todos los cantones ribereños han favorecido acciones en esta actividad. Esto debido a la inexistencia de atractivos idóneos que puedan convertirse en recurso turístico, además de una debilidad latente en la articulación del territorio, los Planes de Desarrollo y Ordenamiento Territorial (PD y OT), pues presentan acciones discordantes, así como una deficiente integración en los instrumentos de planificación territorial e información local.

En otros casos, los centros decisores emprendieron de manera integrada el ordenamiento turístico de playas y atractivos existentes. En este sentido, el Plan Nacional de Competitividad Turística de Ecuador (MINTUR, 2002) dio las condiciones para el desarrollo turístico y eligió un solo cantón (Puerto López) como ejemplo para imitar en idoneidad hacia el resto de cantones costeros. No obstante, ese cantón no tuvo en cuenta el enfoque sostenible desde la multidimensionalidad; de esta manera, lo social y lo político-institucional quedan excluidos. A esto se le suman desajustes y desorden en la actividad turística, así como el incremento no controlado de visitantes. 
Así, el turismo sostenible surge con un dinamismo diferenciado a la mayoría de los sectores, debido a que un consumidor de turismo viaja al productor y al producto. Las consideraciones anteriores conllevan, según Denman (2006), a tres aspectos significativos: interacción, sensibilización y dependencia; aspectos que involucran a visitantes, comunidades anfitrionas. Además, se debe tener en cuenta los problemas ambientales, las diferencias entre naciones y culturas, en relación con la exigencia de los visitantes por entornos naturales más atractivos.

Loacontecidodemuestraclaramentequesedebe asegurar la sostenibilidad mediante un proceso continuo en la gestión. Con respecto a esto, Yunis (2004) expresó que se requiere de mecanismos y de supervisión permanente, además de la ayuda de un sistema de indicadores de sostenibilidad para que se verifique, de manera periódica, el estado medioambiental, social, económico e institucional de los destinos turísticos costeros. No obstante, el visitante debe contribuir, con su presencia y actitud, hacia un desarrollo sostenible para que su viaje sea más saludable.

Ante la situación planteada, la Organisation for Economic Cooperation and Development (OECD, 2013) observa que los turistas son cada día más rigurosos. Ahora son más perceptivos de los problemas ambientales; por lo tanto, los destinos turísticos deben considerar: aspectos socioculturales, patrimoniales, entorno cultural, paisaje urbano. Igualmente, deben observar los procesos del ciclo del agua, la emisión de gases, el gasto energético, debido a que el consumo está cambiando hacia opciones turísticas cada vez más influenciadas por la sostenibilidad.

El autor considera significativo expresar que el turismo sostenible no representa una modalidad turística; por el contrario, todas las modalidades turísticas deben ser sostenibles, donde los actores involucrados en esta actividad se adapten a contextos de diferentes destinos; y así, gestionar esta actividad para la búsqueda del equilibrio anhelado de las dimensiones de la sostenibilidad.

Modelo de gestión del desarrollo turístico sostenible para cantones costeros

El diseño de un modelo debe tener características idóneas para el ámbito de actuación, como adaptarse a la realidad turística y encauzarse a la sostenibilidad. Asimismo, debe implementarse con flexibilidad para adaptarse a las necesidades presentes y futuras derivadas por cambios del entorno. En este sentido, los modelos deben estar estructurados en un conjunto de elementos sustanciales: objetivo, premisas, principios, cualidades, enfoques y momentos fundamentales (Menoya, 2015).

De acuerdo con los aportes de Haddad y Andrade, 1989; Sousa, 2001; Vogt, 2002; Martín, 2006; Machado, 2013; Menoya, 2015, un modelo es la forma lógica para representar, de manera sistemática y simplificada, un determinado proceso. A partir de este enfoque de referencia, se diseña el modelo de gestión para el desarrollo turístico sostenible en cantones costeros del sur de Manabí, Ecuador, como solución al problema en el impulso de esta actividad en los territorios caracterizados.

La gestión turística en Ecuador ha sido el epicentro de buenas iniciativas para el desarrollo de esta actividad. En este sentido, el Ministerio de Turismo (MINTUR, 2015a; 2015b) plantea que Ecuador debe convertir a cada uno de los sitios turísticos en destinos de excelencia; por ello, el autor considera que el modelo propuesto beneficiará de manera significativa la coordinación entre actores. Esta relación permite viabilizar las metas dirigidas en la gestión para el desarrollo turístico sostenible mediante una ágil toma de decisiones. 
Machado (2013), citado por Montesdeoca (2014), determina que la puesta en marcha de un modelo adecuado a un territorio les permitirá a sectores económicos avanzar en un mismo sentido. El autor considera que esto facilita la conservación del hábitat, protege el patrimonio sociocultural y fortalece la sistematización político institucional en los espacios con potencial turístico. Tal es así que el modelo de gestión debe conducir a la integración de los recursos y agentes involucrados, a través de políticas apropiadas al cantón costero que favorezcan, de mejor manera, la toma de decisiones gubernamentales.

La implementación de este modelo constituye una gran oportunidad para el desarrollo turístico sostenible en cantones costeros, debido a su vinculación y articulación entre las políticas económicas, sociales y ambientales, que supone, a la vez, la coyuntura de los medios apropiados para alcanzar las opciones escogidas y se convierta en una herramienta hacia los actores locales de los cantones costeros del sur de la provincia Manabí. Para la construcción conceptual y práctica del modelo, se tuvieron en cuenta los siguientes aspectos:

- De manera fundamental, el modelo está delineado para resolver inconvenientes complejos a través de un proceso armónico integrador de políticas y de participación ciudadana, por eso, se considera como meta el bienestar humano. Asimismo, corresponde al equilibrio entre las dimensiones de la sostenibilidad.

- El modelo de gestión para el desarrollo turístico sostenible para cantones costeros involucra a actores de mayor influencia en la gestión de estos espacios, a saber: Gobierno nacional con los distintos organismos que intervienen en la localidad, gabinetes zonales, Comité Zonal de Turismo, gobierno autónomo descentralizado provincial, gobierno autónomo descentralizado cantonal, sector comunitario.
- El análisis comparativo entre diferentes modelos orientados al desarrollo turístico brindó un soporte teórico al modelo propuesto, más allá del contexto nacional en el cual se basan sus condiciones de implementación.

- Para el modelo, el GAD se constituye en el centro decisor primordial, al ser el cliente con las competencias para planificar el desarrollo local y formular lo correspondiente con los planes de ordenamiento territorial de manera articulada con la planificación nacional, zonal, provincial y cantonal.

- La propuesta se cimenta en una estrategia de desarrollo local que se enmarca en elementos de entrada impulsados por la Secretaria Nacional de Planificación y Desarrollo (SEMPLADES, 2015).

En este contexto y para el desarrollo turístico con enfoque sostenible, los autores de esta propuesta consideran fortalecer factores como empleo, productividad, conocimiento, bienestar social, entre otros. Además, deben reducirse: índices de pobreza, contaminación, deforestación, incapacidad turística.

Con los elementos analizados, se concibe el modelo propuesto en la figura 4, el cual expresa las relaciones y los flujos informativos que se establecen entre componentes que lo conforman, las entradas y las salidas, y brinda una visión sistémica de la gestión para el desarrollo turístico sostenible en cantones costeros del sur de Manabí.

El modelo de gestión para el desarrollo turístico en cantones costeros de la provincia Manabí, desde su entorno, parte de principios, leyes y normas constitucionales, los cuales establecen que la planificación garantizará el ordenamiento territorial y será obligatoria en todos los cantones costeros con disposición turística. Además, el modelo contempla las normas públicas desde el PNBV que promueve impulsar el turismo como 
Modelo de Desarrollo Turístico Sostenible para cantones costeros

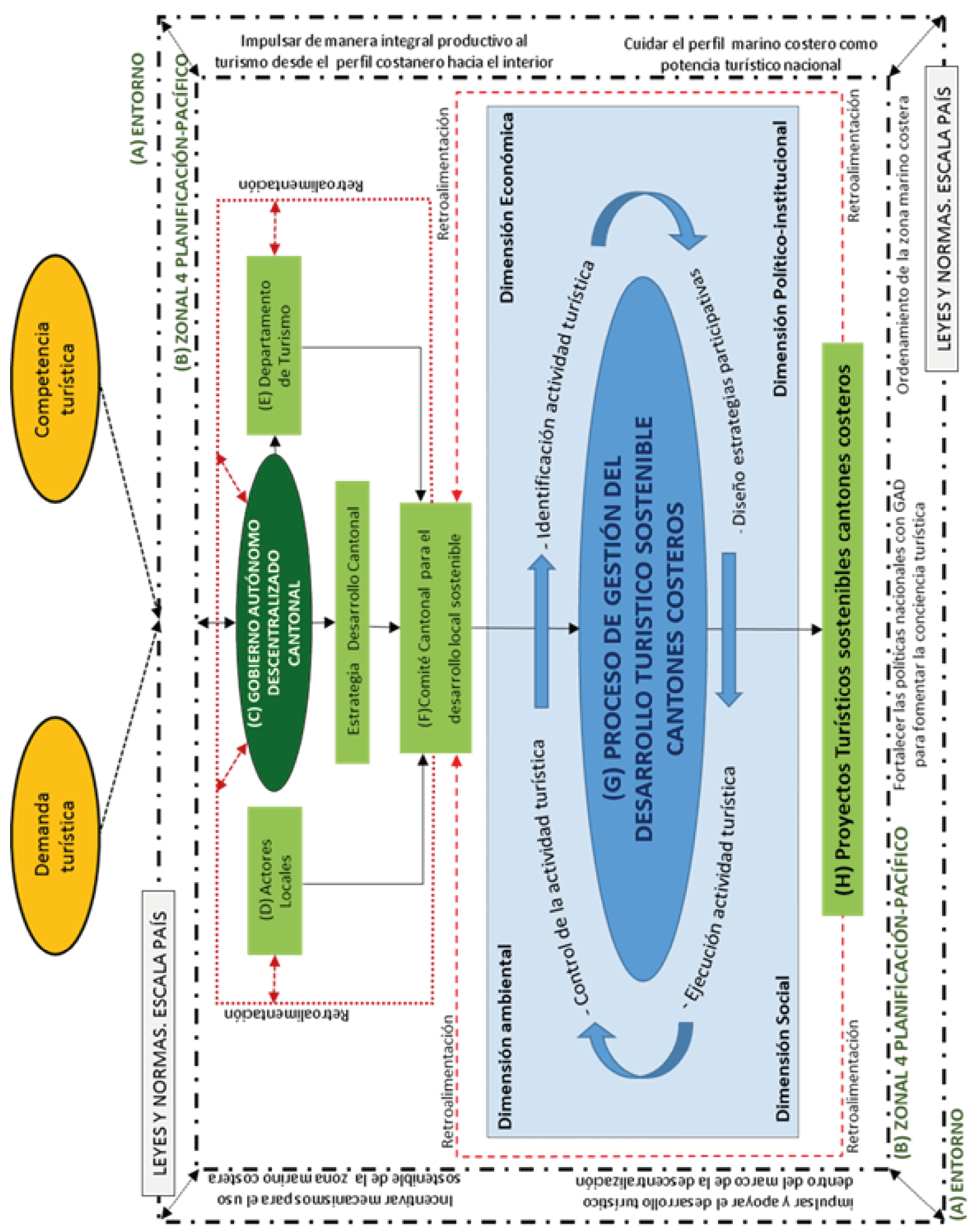

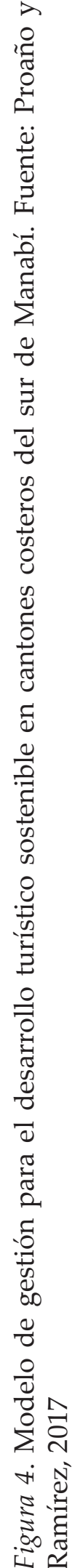


uno de los sectores prioritarios para la atracción de inversión nacional y extranjera.

Entre los soportes perceptibles del modelo que regirán el proceder, están los siguientes: fortalecer las políticas nacionales; fomentar la conciencia turística; ordenar la zona marinocostera; cuidar el perfil costanero como potencial turístico nacional; impulsar, de manera integral, el turismo desde el perfil costanero; apoyar el desarrollo turístico dentro del marco de la descentralización; incentivar mecanismos para el uso sostenible de la zona marino costera.

Para la aplicación del modelo, se deben brindar las siguientes premisas:

- La existencia de mecanismos (convenios) que permitan instrumentar y hacer cumplir la voluntad política para que el GAD y los actores comprendidos en la conformación del comité de desarrollo local sostenible apliquen la metodología que asiste al modelo para su implementación

- El compromiso y la disposición de actores locales públicos, privados y de economía popular y solidaria, que accedan a la necesidad de lograr la gestión para el desarrollo turístico en su localidad. Esta gestióntendráunenfoquedesostenibilidad, además del compromiso de los actores de mayor influencia en la gestión de dichas áreas, para que contribuyan y aprendan de manera permanente en el proceso.

- Los centros decisores deben identificar y sensibilizar a actores locales que sean representativos en la actividad turística. A partir de este momento, deben realizar talleres sobre la necesidad de un turismo sostenible, para que cada uno tenga claridad sobre las dimensiones que se deben conservar y equilibrar. Dicho proceso debe ser acompañado de los líderes, quienes deben fortalecer estos talleres

\section{Conclusión}

A partir de los fundamentos teóricos y metodológicos resultado de la investigación, se detectaron brechas relacionadas con la gestión del desarrollo turístico sostenible. Esto contribuye con la concepción de un modelo para la gestión turística en cantones costeros, que permite mejorar la planificación estratégica de su orientación turística y la toma de decisiones de los actores locales involucrados en el desarrollo propuesto por el país.

El diagnóstico de cantones costeros del sur de Manabí permitió identificar problemáticas en la gestión del desarrollo turístico. Aquí se observa predominio economicista y de comercialización sobre los productos y la actividad turística ejercida, lo cual se aleja de un enfoque sostenible.

Se aplicará el método Delphi para identificar factores positivos y negativos en la gestión turística; además, permitirá validar la hipótesis planteada en la investigación mediante el consenso de expertos.

\section{Referencias}

Alcântara, B. (2014). Relação entre desenvolvimento sustentável e imagem de lugar de um destino turístico: proposição de um modelo estrutural. (Brasil., ed.) Revista Brasileira de Pesquisa em turismo.

Asamblea Constituyente. (2008). Constitución de la República de Ecuador. Montecristi-Ecuador.

Bravo, M.; Bigué, M. \& Vinueza, D. (2016). Plan Nacional de Control y Vigilancia de Áreas Marino Costeras Protegidas de Ecuador Continental. Programa Marino de WildAid, Ministerio de Ambiente MAE.

Calero, A. (1976). Técnicas de muestreo. Vienlo. Habana: Pueblo y Educación.

Denman, R. (2006). Por un turismo más sostenible. Guía para responsables políticos. Madrid: Progra- 
ma de las Naciones Unidas para el Medio Ambiente y Organización Mundial del Turismo.

Haddad, P. \& Andrade, T. (1989). Métodos de análise regional economía regional: teorías e métodos de análise, BNB. (P. R. Haddad, ed.) Fortaleza, 207-223.

Instituto Geográfico Militar (IGM) (2014). Geografía Económica: Recursos, Sectores, Infraestructura. Quito: Autor. Recuperado de www.igm. gob.ec.

Instituto Nacional de Estadística y Censos (INEC, 2010). Censo Población y Vivienda. Quito: Autor.

Instituto Nacional de Estadística y Censos (INEC, 2016). Compendio de estadísticas a nivel Nacional, Provincial y Cantonal 2010-2016. Instituto Nacional de Estadística y Censos-Ecuador. Recuperado de www.ecuadorencifras.gob.ec

Machado, E. (2013). Integración y Diseño del Producto Turístico. GeoGraphos, 4(35), 76.

Ministerio de Ambiente de Ecuador (MAE) (2014). Conservacion de la Biodiversidad Marina y Costera de Ecuador. Guayaquil: Subsecretaría de Gestión Marino Costera del Ministerio de Ambiente.

Ministerio de Ambiente de Ecuador (MAE) (2016). Código Orgánico del Ambiente. Ley Ministerio de Ambiente de Ecuador, Quito-Ecuador.

Martín, R. (2006). Principios, Organización y Práctica del Turismo. Centro de Estudios Turísticos, Universidad de La Habana.

Menoya, S. (2015). Modelo de Gestión del Turismo desde el Gobierno Local para Municipios con Vocación Turística basado en el enfoque de Cadena de Valor. (Tesis presentada en opción al grado científico de doctor en Ciencias Económicas), Pinar del Río-Cuba.

Ministerio de Coordinación de la Política y Gobiernos Autónomos Descentralizados (2011). Código Orgánico de Organización Territorial, Au- tonomía y Descentralización (COOTAD). Quito: V\&M Gráficas.

Ministerio de Turismo (MINTUR) (2002). Plan Nacional de Competitividad Turística. Proyecto OMT-PNUD-ECU, Quito - Ecuador.

Ministerio de Turismo (MINTUR) (2007). Plan estratégico de desarrollo de turismo sostenible para Ecuador. PLANDETUR 2020. Ministerio de Turismo de Ecuador. Quito: MINTUR.

Ministerio de Turismo (MINTUR) (2010). Proyecto PLANDETUR. Ministerio de Turismo de Ecuador. Quito: MINTURr.

Ministerio de Turismo (MINTUR) (2014). Plan Integral de Desarrollo para el Turismo Interno de Ecuador. Subsecretaría de Gestión Turística. Quito:MINTUR.

Ministerio de Turismo (MINTUR) (2015a). Ecotourism and sustainable tourism conference 2015. Viaja Primero Ecuador (6). Quito: MINTUR.

Ministerio de Turismo (MINTUR) (2015b). Ecuador Potencia Turística. Ministerio de Turismo Ecuador. Quito: MINTUR.

Montesdeoca Álva, C. (2014). Plan de Gestión Integrada en el Turismo Rural del Cantón Bolívar: Caso de estudio Parroquia Membrillo. (Tesis, Escuela Politécnica Agropecuaria de Manabí, Calceta), Manabí-Ecuador.

Organisation for Economic Co-operation and Development (OECD) (2013). The Organisation for Economic Co-operation and Development. Green Innovation in Tourism Services. Recuperado de: http://dx.doi.org/10.1787/5k4bxkt1cjd2-en

Plan de Desarrollo y Ordenamiento Territorial Manta (PDyOT Manta) (2016). Manta. Plan de Desarrollo y Ordenamiento Territorial del Cantón Manta. Manta-Manabí-Ecuador.

Plan de Desarrollo y Ordenamiento Territorial Jaramijó (PDyOT Jaramijó) (2016). Jaramijó. 
Plan de Desarrollo y Ordenamiento Territorial del Cantón Jaramijó. Jaramijó-Ecuador.

Plan de Desarrollo y Ordenamiento Territorial Montecristi (PDyOT Montecristi) (2016). Plan de Desarrollo y Ordenamiento Territorial del Cantón Montecristi. Montecristi-Manabí-Ecuador.

Pérez, I. (2013). Relaciones de actores sociales en el ecoturismo como base al desarrollo territorial en el parque nacional Viñales y su area de influencia, Cuba. La Habana: Universidad de la Habana.

Plan Nacional paara el Buen Vivir (PNBV) (2013). Plan Nacional de Desarrollo/Plan Nacional para el Buen Vivir 2013-2017. Quito: SENPLADES, Secretaría Nacional de Planificación y Desarrollo.

PRO ECUADOR. (2014). Perfil Sectorial de Turismo para el Inversionista. Instituto de Promoción de Exportaciones e Inversiones, Quito-Ecuador.

Secretaría Nacional de Planificación y Desarrollo (SENPLADES) (2010). Propuestas de Desarrollo y Lineamientos para el Ordenamien- to Territorial. Secretaría Nacional de Planificación y Desarrollo. Quito: Imprenta Monsalve Moreno.

Secretaría Nacional de Planificación y Desarrollo (SENPLADES) (2015). Agenda Zonal 4-Pacífico, Manabí y Santo Domingo de los Tsáchilas. Secretaría Nacional de Planificación y Desarrollo. Quito: ( ) Senplades.

Secretaría Técnica del Mar (SETEMAR) (2014). Política de desarrollo del territorio marino costero. Secretaría Técnica del Mar, Manta-Ecuador.

Sousa, R. (2001). Análisis en la creación de valor en las ganancias de viajes. (Tesis Doctoral, Universidad Complutense de Madrid), Madrid-España.

Vogt, C. (2002). Modelos e modelagens, Comciência. eletrônica de jornalismo.

Yunis, E. (2004). El imperativo de la sostenibilidad en el turismo del siglo XXI. Recuperado de http://fama2.us.es:8080/turismo/turismonet1/ economia $\% 20 \mathrm{del} \% 20$ turismo/tuismo\%20sostenible/imperativo $\% 20 \mathrm{de} \% 201 \mathrm{a} \% 20$ sostenibilidad\%20en\%20el\%20tu.

Recibido: 06 de octubre de 2017 Aceptado: 05 de noviembre de 2017 